\title{
RECURRENT TENSORS ON A LINEARLY CONNECTED DIFFERENTIABLE MANIFOLD ${ }^{(1)}$
}

\author{
BY \\ YUNG-CHOW WONG
}

Introduction. Let $M$ be a connected smooth (i.e., $C^{\infty}$ ) manifold of dimension $n$, and $B$ the total space of the frame bundle over $M$. Let a linear connection be given on $M$, and let $B\left[z_{0}\right]$ be the submanifold of $B$ consisting of all the points which can be joined to a given point $z_{0}$ in $B$ by sectionally smooth horizontal curves.

The purpose of this paper is to elaborate on a natural correspondence set up by S. S. Chern [2, pp. 78-79] between tensors of type $(r, s)$ on $M$ and sets of $n^{r+s}$ functions of a particular type on $B$, and use it to prove the theorem that $a$ tensor $S$ on $M$ is recurrent (i.e., $S$ is not a zero tensor and its covariant derivative is equal to the tensor product of a covariant vector and $S$ itself) iff the restrictions to $B\left[z_{0}\right]$ of its corresponding functions on $B$ have no common zero and are proportional to a set of constants. We give several applications of this theorem, obtaining among others the following results:

(i) A recurrent tensor on $M$ has no zero (Theorem 3.8);

(ii) A property obtained by K. Nomizu [4, p. 73] characterizing linear connections with covariantly constant curvature tensor or covariantly constant torsion tensor (Theorem 4.3);

(iii) A property characterizing linear connections with recurrent curvature or recurrent torsion similar to (ii) above (Theorem 4.2);

(iv) The holonomy group of a linear connection with recurrent curvature is at most of dimension $n(n-1) / 2$ (Corollary 4.4).

The author is deeply indebted to Professor S. S. Chern for his generous help during the first half of the year 1959 at the University of Chicago when the author tried to acquaint himself with the modern theory of linear connections. The author is also grateful to the referee for pointing out a gap in the original proof of our main theorem (3.9) and for his valuable suggestions which have resulted in several improvements in this paper.

1.1. Bundle of frames. In $\S \S 1.1-1.5$, we summarize some of the results on linear connections which are needed for our later work. We assume as known the classical theory of linear connections and the elementary properties of $n$-dimensional smooth manifolds (class $C^{\infty}$ and with countable base). All the vector fields, tensor fields, $q$-forms, etc. defined on a smooth manifold or on part of it are assumed to be of class $C^{\infty}$ unless stated otherwise. Each of the

Presented to the Society, September 2, 1960 under the title Recurrent tensors on a differentiable manifold; received by the editors June 22, 1960 and, in revised form, November 15, 1960.

(1) To the University of Hong Kong on its Golden Jubilee, 1961. 
indices $i, j, k, \cdots ; \alpha, \beta, \gamma, \cdots$ runs from 1 to $n$. Latin indices have tensor character except in the case of local coordinates, while Greek indices are merely used to distinguish one function or tensor from another function or tensor of the same type. Summation over repeated indices, Latin or Greek, is implied.

Let $M$ be a smooth manifold of dimension $n$. A frame $z(u)$ in $M$ is composed of a point $u \in M$ and an ordered set of $n$ linearly independent tangent vectors at $u$. Let $B(M)$ denote the collection $(B, M, \pi, F=G, G)$, where

$B$, dimension $n+n^{2}$, is the space of all frames in $M$;

$M$, dimension $n$, is the given smooth manifold;

$\pi$ is the projection $B \rightarrow M$ defined by $z(u) \rightarrow u$;

$F$, the standard fiber, is the space of invertible $n \times n$ matrices;

$G=G L(n, R)$ is the real general linear group which we may identify with $F$. Provided with a natural topology and differential structure, $B$ becomes a smooth manifold and $B(M)$ a principal fiber bundle over $M$. This $B(M)$ is the frame bundle over $M$.

Let $\left(U, u^{i}\right)$ be a covering of $M$ by coordinate neighborhoods $U$ and local coordinates $u^{i}$. Then in $U, \partial / \partial u^{i}$ form a basis of vector fields, and $d u^{i}$ a basis of 1 -forms dual to the basis $\partial / \partial u^{i}$. If $z(u)$ is a frame at $u \in U$, the tangent vectors $X_{\alpha}(u)$ of $z(u)$ can be expressed as

$$
X_{\alpha}(u)=x_{\alpha}^{i}\left(\frac{\partial}{\partial u^{i}}\right)_{u},
$$

where $x_{\alpha}^{i}$ are $n^{2}$ real numbers such that $\operatorname{det}\left(x_{\alpha}^{i}\right) \neq 0$. Thus, $\left\{\pi^{-1}(U),\left(u^{i}, x_{\alpha}^{i}\right)\right\}$ form a covering of $B$ by coordinate neighborhoods $\pi^{-1}(U)$ and local coordinates $\left(u^{i}, x_{\alpha}^{i}\right)$.

If $\left(U, u^{i}\right)$ and $\left(U^{*}, u^{i}\right)$ are two coordinate systems in $M$, and $u \in U \cap U^{*}$, then

$$
u^{i^{*}}=u^{i^{*}}\left(u^{1}, \cdots, u^{n}\right) .
$$

If $z(u) \in \pi^{-1}\left(U \cap U^{*}\right)=\pi^{-1}(U) \cap \pi^{-1}\left(U^{*}\right)$ has local coordinates $\left(u^{i}, x_{\alpha}^{i}\right)$ and $\left(u^{i^{*}}, x_{\alpha}^{i^{*}}\right)$, then

$$
x_{\alpha}^{i^{*}}=x_{\alpha}^{k} \frac{\partial u^{i^{*}}}{\partial u^{k}}, \text { or in matrix form, } x^{*}=x J(u) .
$$

Thus, the transformation of coordinates in $\pi^{-1}\left(U \cap U^{*}\right)$ is expressed by equations (1.2) and (1.3).

The action of $G$ on $B$ (to the right)

$$
z \rightarrow R_{a} z=z a, \text { where } z \in B, a \in G,
$$

is defined as follows: If $z$ is the frame in $M$ consisting of the $n$ vectors $X_{\alpha}$ at $u \in M$, then $R_{a} z=z a$ is the frame in $M$ consisting of the $n$ vectors $a_{\alpha^{\prime}}^{\alpha} X_{\alpha}$ at 
$u \in M$, where $a=\left(a_{\alpha^{\prime}}^{\alpha}\right)$. Expressed in terms of local coordinates in $B$, the action of $G$ on $B$ is

$$
a:\left(u^{i}, x_{\alpha}^{i}\right) \rightarrow\left(u^{i}, a_{\alpha^{\prime}}^{\alpha} x_{\alpha}^{i}\right) .
$$

1.2. Linear connections on $M$ (cf. Chern [2, Chapter 4]). A linear connection on $M$ is an assignment to each coordinate system $\left(U, u^{i}\right)$ in $M$ of an $n \times n$ matrix $\gamma$ of 1 -forms $\gamma_{k}^{q}$ such that, if $U \cap U^{*} \neq \square$, the matrices $\gamma$ and $\gamma^{*}$ assigned to $\left(U, u^{i}\right)$ and $\left(U^{*}, u^{i}\right)$ and related by

$$
\gamma=d J \cdot J^{-1}+J \gamma^{*} J^{-1}, \text { where } J=\left(\frac{\partial u^{i^{*}}}{\partial u^{k}}\right) .
$$

When we write $\gamma_{k}^{i}=\Gamma_{j k}^{i} d u^{j}$, (1.5) gives the usual transformation law for the components $\Gamma_{j k}^{i}$ of a linear connection.

Let us denote by $d u$ the $1 \times n$ matrix $\left(d u^{i}\right)$, by $x$ the $n \times n$ matrix $\left(x_{\alpha}^{i}\right)$, by $x^{-1}$ the inverse $\left(x_{i}^{\alpha}\right)$ of $x$, and by $d x$ the $n \times n$ matrix $\left(d x_{\alpha}^{i}\right)$. Then we have: (1.6) The $n 1$-forms $d u \cdot x^{-1}$, defined in each coordinate neighborhood $\pi^{-1}(U)$ in $B$, piece together to form $n 1$-forms $\theta=\left(\theta^{\alpha}\right)$ globally defined on $B$.

(1.7) If a linear connection $\gamma$ on $M$ is given, the $n^{2} 1$-forms $d x \cdot x^{-1}+x \gamma x^{-1}$, defined in each coordinate neighborhood $\pi^{-1}(U)$ in $B$, piece together to form $n^{2} 1$-forms $\omega=\left(\omega_{\mu}^{\lambda}\right)$ globally defined on $B$.

(1.8) The $n+n^{2} 1$-forms $\theta$ and $\omega$ are everywhere linearly independent on $B$.

(1.9) Under the action $R_{a}: z \rightarrow z a$ of $G$ on $B$,

$$
R_{a}^{*} \theta_{z a}=\theta_{z} a^{-1}, \quad R_{a}^{*} \omega_{z a}=a \omega_{z} a^{-1}
$$

where $R_{a}^{*}$ denotes the mapping on differential forms on $B$ induced from $R_{a}$, and the right sides of the equations are products of the matrices $a, a^{-1}, \theta_{2}, \omega_{z}$.

A tangent vector $Z$ in $B$ is vertical if it annihilates all the 1 -forms $\theta^{\alpha}$, i.e., if $\left\langle\theta^{\alpha}, Z\right\rangle=0$, where $\left\langle\theta^{\alpha}, Z\right\rangle$ denotes the value of the 1 -form $\theta^{\alpha}$ at the vector $Z$. The tangent $n^{2}$-planes to the fibers in $B$ form a field of vertical $n^{2}$-planes on $B$. On the other hand, a tangent vector $Z$ in $B$ is horizontal if it annihilates all the $n^{2} 1$-forms $\omega_{\mu}^{\lambda}$. The equations $\omega_{\mu}^{\lambda}=0$ define the field of horizontal $n$-planes on $B$. Then we have:

(1.10) At each point of $B$, the vertical $n^{2}$-plane and the horizontal $n$-plane are complementary. And the field of vertical $n^{2}$-planes and the field of horizontal $n$-planes are both invariant under the action of $G$ on $B$.

(1.11) Conversely, if a field of $n$-planes is given on $B$ which is complementary to the field of vertical $n^{2}$-planes and which is invariant under the action of $G=G L(n, R)$ on $B$, then there exists on $M$ a unique linear connection for which the field of horizontal $n$-planes is the given field of $n$ planes.

1.3. Horizontal curves, lifts, and the submanifold $B\left[z_{0}\right]$. A sectionally 
smooth curve (with a finite number of smooth sections) in $B$ is called horizontal if each of its tangent vectors whenever it is defined is horizontal. Let $u(\tau), 0 \leqq \tau \leqq 1$, be any sectionally smooth curve in $M$. If $z(\tau)$ is a sectionally smooth horizontal curve in $B$ such that $u(\tau)=\pi z(\tau)$, then $z(\tau)$ is called a lift of $u(\tau)$.

(1.12) If $z(\tau)$ is a lift of any sectionally smooth curve $u(\tau)$ in $M$, then $R_{a} z(\tau)$, for any $a \in G$, is also a lift of $u(\tau)$.

(1.13) Given any sectionally smooth curve $u(\tau), 0 \leqq \tau \leqq 1$, in $M$ and any point $z_{0} \in B$ such that $\pi z_{0}=u(0)$, then there exists a unique lift $z(\tau)$ of $u(\tau)$ in $B$ such that $z(0)=z_{0}$.

Let $z_{0}, z$ be any two points in $B$. Whenever there exists a sectionally smooth horizontal curve in $B$ joining $z_{0}$ to $z$, we write $z \sim z_{0}$. Obviously the relation $\sim$ is an equivalence relation in the set of points of $B$. If $z \sim z_{0}$, then $z a \sim z_{0} a$ for any $a \in G$.

The set of elements $a \in G$ such that $z_{0} a \sim z_{0}$ form a subgroup of $G$ which is called the holonomy group $\Phi_{z_{0}}$ (of the linear connection) with reference point $z_{0}$.

Let $B\left[z_{0}\right]=\left\{z \mid z \in B, z \sim z_{0}\right\}$. Then Ambrose and Singer [1] have proved that $B\left[z_{0}\right]$ is a regular submanifold of $B$ which, in the case of $M$ being connected, can be regarded as a reduced bundle of $B(M)$ with structural group $\Phi_{z_{0}}$. It follows from definition that any two points of $B\left[z_{0}\right]$ can be joined by a sectionally smooth horizontal curve. This submanifold of $B\left[z_{0}\right]$ will play an important role in our results.

1.4. Torsion forms and curvature forms (cf. Chern [2, Chapter 4]). (1.14) If we put

$$
\Theta=d \theta-\theta \wedge \omega, \quad \Omega=d \omega-\omega \wedge \omega,
$$

then $\Theta=\left(\Theta^{\alpha}\right), \Omega=\left(\Omega_{\mu}^{\lambda}\right)$ are $n+n^{2} 2$-forms on $B$ which can be expressed as

$$
\Theta^{\gamma}=(1 / 2) T_{\alpha \beta}^{\gamma} \theta^{\alpha} \wedge \theta^{\beta}, \quad \Omega_{\mu}^{\lambda}=(1 / 2) R_{\mu \alpha \beta}^{\lambda} \theta^{\alpha} \wedge \theta^{\beta},
$$

where $T_{\alpha \beta}^{\gamma}, R_{\mu \alpha \beta}^{\gamma}$ are functions on $B$.

(1.15) In local coordinates $\left(u^{i}, x_{\alpha}^{i}\right)$,

$$
\begin{aligned}
\Theta & =-d u \wedge \gamma x^{-1}, & \Omega & =x(d \gamma-\gamma \wedge \gamma) x^{-1} ; \\
T_{\alpha \beta}^{\gamma} & =T_{j k}^{i} x_{i}^{\gamma} x_{\alpha}^{j} x_{\beta}^{k}, & R_{\mu \alpha \beta}^{\lambda} & =R_{j k l}^{i} x_{i}^{\lambda} x_{\mu}^{j} x_{\alpha}^{k} x_{\beta}^{l} ; \\
T_{j k}^{i} & =\Gamma_{j k}^{i}-\Gamma_{k j}^{i}, & R_{j k l}^{i} & =\frac{\partial \Gamma_{l j}^{i}}{\partial u^{k}}-\frac{\partial \Gamma_{k j}^{i}}{\partial u^{l}}+\Gamma_{k h}^{i} \Gamma_{l j}^{h}-\Gamma_{l h}^{i} \Gamma_{k j}^{h} .
\end{aligned}
$$

The 2-forms $\Theta, \Omega$ on $B$ are the torsion forms and the curvature forms of the linear connection $\gamma$ on $M$. We observe that $T_{\alpha \beta}^{\gamma}, R_{\mu \alpha \beta}^{\lambda}$ are functions on $B$, while $T_{j k}^{i}, R_{j k l}^{i}$ are the components, in the coordinate system $\left(U, u^{i}\right)$ in $M$, of the torsion tensor and the curvature tensor of the linear connection $\gamma$ with components $\Gamma_{j k}^{i}$ in $\left(U, u^{i}\right)$. 
1.5. Fundamental vector fields and basic vector fields. Let $E_{\lambda}^{\mu}, E_{\alpha}$ be the $n^{2}+n$ vector fields on $B$ which are dual to the $n+n^{2} 1$-forms $\theta^{\beta}, \omega_{\sigma}^{\rho}$, so that

$$
\begin{array}{ll}
\left\langle\theta^{\beta}, E_{\alpha}\right\rangle=\delta_{\alpha}^{\beta}, & \left\langle\omega_{\sigma}^{\rho}, E_{\alpha}\right\rangle=0, \\
\left\langle\theta^{\beta}, E_{\lambda}^{\mu}\right\rangle=0, & \left\langle\omega_{\sigma}^{\rho}, E_{\lambda}^{\mu}\right\rangle=\delta_{\lambda}^{\rho} \delta_{\sigma}^{\mu} .
\end{array}
$$

The vector fields $E_{\lambda}^{\mu}, E_{\alpha}$ have the following local expressions:

$$
E_{\lambda}^{\mu}=x_{\lambda}^{j} \frac{\partial}{\partial x_{\mu}^{j}}, \quad E_{\alpha}=x_{\alpha}^{j}\left(\frac{\partial}{\partial u^{j}}-\Gamma_{j k}^{i} x_{\gamma}^{k} \frac{\partial}{\partial x_{\gamma}^{i}}\right) .
$$

We call $c_{\mu}^{\lambda} E_{\lambda}^{\mu}$ ( $c_{\mu}^{\lambda}$ constant) a fundamental vector field, and $c^{\alpha} E_{\alpha}$ ( $c^{\alpha}$ constant) a basic vector fueld. They are identical with the vector fields of the same names defined by Ambrose-Singer [1] and Nomizu [4, p. 49].

The structure equations of the linear connection $\gamma$ can be expressed in either of the following two equivalent forms:

$$
\begin{aligned}
d \theta^{\gamma}=\theta^{\alpha} \wedge \omega_{\alpha}^{\gamma}+(1 / 2) T_{\alpha \beta}^{\gamma} \theta^{\alpha} \wedge \theta^{\beta}, \quad d \omega_{\mu}^{\lambda}=\omega_{\mu}^{\rho} \wedge \omega_{\rho}^{\lambda}+(1 / 2) R_{\mu \alpha \beta}^{\lambda} \theta^{\alpha} \wedge \theta^{\beta} \\
{\left[E_{\lambda}^{\mu}, E_{\rho}^{\sigma}\right]=\delta_{\rho}^{\mu} E_{\lambda}^{\sigma}-\delta_{\lambda}^{\sigma} E_{\rho}^{\mu}, } \\
{\left[E_{\alpha}, E_{\lambda}^{\mu}\right]=-\delta_{\alpha}^{\mu} E_{\lambda}, } \\
{\left[E_{\alpha}, E_{\beta}\right]=-T_{\alpha \beta}^{\gamma} E_{\gamma}-R_{\mu \alpha \beta}^{\lambda} E_{\lambda}^{\mu}, }
\end{aligned}
$$

where [ , ] denotes the Lie product of two vector fields. For a discussion of the implications of the structure equations (1.19), see Wong [6].

2.1. Correspondence between tensors on $M$ and functions on $B$. We denote the local coordinates of a point $z \in B$ by $\left\{u^{i}(z), x_{\alpha}^{i}(z)\right\}$. Under the action of $G$ on $B$, the point $z$ is carried by $a \in G$ into the point $z a$ with local coordinates $\left\{u^{i}(z a), x_{\alpha^{\prime}}^{i}(z a)\right\}$. If we put

$$
a=\left(\begin{array}{c}
\alpha \\
\alpha^{\prime}
\end{array}\right), \quad\left(\tilde{a}_{\alpha}^{\alpha^{\prime}}\right)=\text { inverse of the matrix }\left(a_{\alpha^{\prime}}^{\alpha}\right),
$$

then by (1.4),

$$
u^{i}(z a)=u^{i}(z), \quad x_{\alpha^{\prime}}^{i}(z a)=a_{\alpha^{\prime}}^{\alpha} x_{\alpha}^{i}(z), \quad x_{i}^{\alpha^{\prime}}(z a)=x_{i}^{\alpha}(z) \tilde{a}_{\alpha}^{\alpha^{\prime}} .
$$

Any function $f$ on $M$ gives rise to a function $g$ on $B$ defined by $g=f \cdot \pi$. This function $g$ on $B$ has the property that

$$
g(z a)=g(z) \quad \text { for every } a \in G, z \in B .
$$


Conversely, any function $g$ on $B$ having the property (2.3) induces a function $f$ on $M$ defined by $f(u)=g(z)$, where $z$ is any point in $\pi^{-1}(u) \subset B$. Obviously, condition (2.3) is equivalent to that when $g$ is locally expressed in terms of the coordinates $x_{\alpha}^{i}, u^{i}$, it contains only the $u^{i}$.

We shall now set up a correspondence between a tensor $S$ of type $(r, s)$ on $M$ and a set of $n^{r+s}$ functions of certain type on $B$. For convenience, we shall state and prove our result for a tensor of type $(2,1)$, but the result and the proof can be extended in an obvious manner to a tensor of any type.

(2.4) Theorem. To a tensor $S$ of type $(2,1)$ on $M$, there corresponds a set of $n^{3}$ functions $S_{\gamma}^{\alpha \beta}$ on $B$ such that for any $z \in B$ and any $a \in G$,

$$
S_{\gamma^{\prime}}^{\alpha^{\prime} \beta^{\prime}}(z a)=S_{\gamma}^{\alpha \beta}(z) \tilde{a}_{\alpha}^{\alpha^{\prime}} \tilde{a}_{\beta}^{\beta^{\prime}} a_{\gamma^{\prime}}^{\gamma},
$$

where $\left(a_{\alpha}^{\alpha^{\prime}}\right)=a,\left(\tilde{a}_{\alpha^{\prime}}^{\alpha}\right)=$ inverse of the matrix $\left(a_{\alpha^{\prime}}^{\alpha}\right)$. Conversely, to any such set of $n^{2}$ functions on $B$, there corresponds a tensor of type $(2,1)$ on $M$.

If $\left(U, u^{i}\right)$ and $\left\{\pi^{-1}(U),\left(u^{i}, x_{\alpha}^{i}\right)\right\}$ are respectively local coordinate systems in $M$ and $B$ and $S_{\boldsymbol{k}}^{i j}$ are the components in $\left(U, u^{i}\right)$ of the tensor $S$, then the above correspondence is defined locally by [2, pp. 78-79]

$$
S_{\gamma}^{\alpha \beta}=S_{k}^{i j} x_{i}^{\alpha \beta} x_{j} x_{\gamma}^{k}
$$

and is one-to-one.

Proof. First we show that each of the functions $S_{\gamma}^{\alpha \beta}$, defined by (2.6) on every $\pi^{-1}(U)$, is a function on $B$. In fact, in $\pi^{-1}\left(U \cap U^{*}\right) \subset B$, the local coordinates $\left(u^{i}, x_{\alpha}^{i}\right)$ and $\left(u^{i^{*}}, x_{\alpha}^{i^{*}}\right)$ are related by

$$
\begin{aligned}
& u^{i^{*}}=u^{i^{*}}\left(u^{1}, \cdots, u^{n}\right), \\
& \dot{x_{\alpha}^{*}}=x_{\alpha}^{i} \frac{\partial u^{i^{*}}}{\partial u^{i}} \quad\left(\text { i.e., } x_{i^{*}}^{\alpha}=\frac{\partial u^{i}}{\partial u^{i *}} x_{i}^{\alpha}\right) .
\end{aligned}
$$

Since $S$ is a tensor of type $(2,1)$ on $M$,

$$
S_{k^{*}}^{i^{*} *^{*}}=S_{k}^{i j} \frac{\partial u^{i *}}{\partial u^{i}} \frac{\partial u^{i^{*}}}{\partial u^{j}} \frac{\partial u^{k}}{\partial u^{k^{*}}} .
$$

So it follows at once from (2.6-2.8) that

$$
S_{k^{*}}^{i^{*} x_{i}^{*} x_{j^{*}}^{\alpha} x_{\gamma}^{k^{*}}}=S_{k}^{i j} x_{i}^{\alpha} x_{j}^{\beta} x_{\gamma}
$$

Hence the functions $S_{\gamma}^{\alpha \beta}$ defined locally by (2.6) is defined globally on $B$. That they satisfy the conditions (2.5) is an immediate consequence of (2.2).

Conversely, let $S_{\gamma}^{\alpha \beta}$ be a set of $n^{3}$ functions on $B$ satisfying the conditions (2.5). On each $\pi^{-1}(U)$, define the functions $S_{\mathbf{k}}^{1 y}$ by 


$$
S_{k}^{i j}=S_{\gamma}^{\alpha \beta} x_{\alpha}^{i} x_{\beta}^{j} x_{k}^{\gamma} .
$$

Using (2.2), we easily verify that $S_{k}^{i j}(z a)=S_{k}^{\text {yj }}(z)$. Thus, the functions $S_{k}^{\text {ij }}$ on $\pi^{-1}(U)$ defined by (2.9) induce functions on $U \subset M$ which we still denote by $S_{\mathbf{k}}^{1 j}$.

Finally, we show that these functions $S_{k}^{i j}$ on $U$ are components in $\left(U, u^{i}\right)$ of a tensor of type $(2,1)$ on $M$. Let $\pi^{-1}\left(U^{*}\right)$ be another coordinate neighborhood in $B$. Then by definition

$$
S_{k^{*}}^{i^{*}}=S_{\gamma}^{\alpha \beta} x_{\alpha}^{*} x_{\beta}^{*} x_{k^{*}}^{j^{*}},
$$

which also induce functions on $U^{*} \subset M$. If $U \cap U^{*} \neq \square$, then on $U \cap U^{*}$,

$$
\begin{aligned}
S_{k^{*}}^{i^{*}} & =S_{\gamma}^{\alpha \beta} x_{\alpha}^{i^{*} x_{\beta}^{*} x_{k^{*}}^{\gamma}} \\
& =S_{\gamma}^{\alpha \beta}\left(x_{\alpha}^{i} \frac{\partial u^{i *}}{\partial u^{i}}\right)\left(x_{\beta}^{j} \frac{\partial u^{j *}}{\partial u^{j}}\right)\left(\frac{\partial u^{k}}{\partial u^{k *}} x_{k}^{\gamma}\right) \\
& =S_{k}^{i j} \frac{\partial u^{i *}}{\partial u^{i}} \frac{\partial u^{j^{*}}}{\partial u^{j}} \frac{\partial u^{k}}{\partial u^{k *}} .
\end{aligned}
$$

This completes the proof of the theorem.

REMARK( $\left.{ }^{2}\right)$. An intrinsic definition of the set of functions $S_{\gamma}^{\alpha \beta} \ldots$ on $B$ which corresponds to a given tensor $S$ on $M$ can also be given. Namely, let $V_{n}$ be a real $n$-dimensional vector space with a fixed basis $\left(e_{1}, \cdots, e_{n}\right)$, which we regard as the standard fiber of the tangent bundle over $M$. Every element $z=\left(X_{1}, \cdots, X_{n}\right)$ of $B$ gives rise to a linear isomorphism of $V_{n}$ onto the tangent space $T_{u}(M)$ which maps each $e_{i}$ upon $X_{i}$. The inverse mapping $z^{-1}$ can be extended to an isomorphism of the tensor algebra over $T_{u}(M)$ onto the tensor algebra over $V_{n}$. For any tensor (field) $S$, say of type $(2,1)$, on $M$, we may define $S_{\gamma}^{\alpha \beta}(z), z \in B$, to be the components of the tensor $z^{-1} S_{x(z)}$ (tensor over $V_{n}$ ) with respect to the basis $\left(e_{i}\right)$. Then formula (2.5) is exactly the transformation between the tensors $z^{-1} \cdot S_{\pi(z)}$ and $(z a)^{-1} \cdot S_{\pi(z)}=a^{-1}\left(z^{-1} S_{\pi(z)}\right)$, where $a^{-1}$ is the automorphism of the tensor algebra over $V_{n}$ which extends the linear transformations $a^{-1}$ of $V_{n}$.

2.2. Horizontal part and absolute differential of a $q$-form on $B$. We now assume that a linear connection $\gamma$ is given on $M$. Since $\theta^{\alpha}, \omega_{\mu}^{\lambda}$ form a basis of 1 -forms on $B$, any differential $q$-form $\phi$ on $B$ can be expressed as

$$
\phi=S_{\alpha_{1}}^{\mu_{1} \cdots \mu_{r} \lambda_{1} \cdots \lambda_{s}} \theta^{\alpha_{1}} \wedge \cdots \wedge \theta^{\alpha_{r}} \wedge \omega_{\mu_{1}}^{\lambda_{1}} \wedge \cdots \wedge \omega_{\mu_{s}}^{\lambda_{g}} \quad r+s=q,
$$

where the $S$ 's are functions on $B$. The horizontal part $h \phi$ of $\phi$ is the differential $q$-form which is the sum of those terms on the right of (2.10) containing $\theta^{\alpha}$ 's alone. $\phi$ is called horizontal if $\phi=h \phi$. For any differential form (or func-

(2) The author owes the observation in this remark to the referee. 
tion) $\phi$ on $B$, the absolute differential $D \phi$ of $\phi$ is the horizontal part of the exterior differential of $\phi$, i.e., $D \phi=h(d \phi)$. For example, we have from (1.14) that $D \theta=\Theta, D \omega=\Omega$.

2.3. Functions on $B$ which correspond to the covariant derivative of a tensor on $M$. Assume that a linear connection $\gamma$ is given on $M$. If $S$ is a tensor of type $(r, s)$ on $M$, the covariant derivative $\nabla S$ of $S$ is a tensor of type $(r, s+1)$ on $M$. We shall now find the set of $n^{r+s+1}$ functions on $B$ which corresponds to $\nabla S$. For convenience, we again take a tensor of type $(2,1)$ on $M$. Then the set of $n^{3}$ functions $S_{\gamma}^{\alpha \beta}$ on $B$ corresponding to $S$ is expressed locally by

$$
S_{\gamma}^{\alpha \beta}=S_{k}^{i j} x_{i}^{\alpha \beta} x_{j}^{\beta} x_{\gamma}^{k}
$$

Taking the differentials of the two sides of this equation and making use of (cf. (1.7))

$$
d x=\omega x-x \gamma, \quad d x^{-1}=\gamma x^{-1}-x^{-1} \omega,
$$

we easily deduce that

$$
D S_{\gamma}^{\alpha \beta}=\left(\delta S_{k}^{i j}\right) \underset{x_{i} x_{j} x_{\gamma}}{\alpha k}
$$

where

$$
\begin{aligned}
\delta S_{k}^{i j} & =d S_{k}^{i j}+\gamma_{i}^{i} S_{k}^{l j}+\gamma^{j} S_{k}^{i l}-\gamma_{k}^{l} S_{l}^{i j}, \\
D S_{\gamma}^{\alpha \beta} & =d S_{\gamma}^{\alpha \beta}+\omega_{\epsilon}^{\alpha} S_{\gamma}^{\epsilon \beta}+\omega_{\epsilon}^{\beta} S_{\gamma}^{\alpha e}-\omega_{\gamma}^{e} S_{\epsilon}^{\alpha \beta} .
\end{aligned}
$$

Observe that the 1 -forms $\delta S_{k}^{\text {ij }}$ on $U$ are the components in $\left(U, u^{i}\right)$ of the covariant differential of the tensor $S$. Therefore, on account of (2.11), the 1forms $D S_{\gamma}^{\alpha \beta}$ on $B$ are the horizontal parts of $d S_{\gamma}^{\alpha \beta}$. (This justifies the notation $D S_{\gamma}^{\alpha \beta}$.) Hence, we can put

$$
\delta S_{k}^{i j}=\left(\nabla S_{k}^{i j}\right) d u^{2}, \quad D S_{\gamma}^{\alpha \beta}=\left(D_{s} S_{\gamma}^{\alpha \beta}\right) \theta^{\bullet},
$$

and (2.11) can be written as

$$
D_{\epsilon} S_{\gamma}^{\alpha \beta}=\left(\nabla S_{k}^{i j}\right) x_{\epsilon}^{l} x_{i}^{\alpha \beta} x_{j}^{\beta} x_{\gamma}^{k} .
$$

Summarising our results, we have

(2.15) ThEOREM. If $S_{\gamma}^{\alpha \beta}$ are the $n^{3}$ functions on $B$ corresponding to a tensor $S$ of type $(2,1)$ on $M$, then the absolute differentials $D S_{\gamma}^{\alpha \beta}=h d S_{\gamma}^{\alpha \beta}$ are related to the covariant differential $\delta S$ by

$$
D S_{\gamma}^{\alpha \beta}=\left(\delta S_{k}^{i j}\right) x_{i}^{\alpha x_{j}^{\beta} x_{\gamma}} .
$$

Furthermore, the functions $D . S_{\gamma}^{\alpha \beta}$ on $B$ defined by 


$$
D S_{\gamma}^{\alpha \beta}=\left(D_{\epsilon} S_{\gamma}^{\alpha \beta}\right) \theta^{\epsilon}
$$

are the $n^{4}$ functions corresponding to the covariant derivative $\nabla S$.

Since all the 1 -forms $\omega_{\mu}^{\lambda}$ vanish on any horizontal curve in $B$, it follows from (2.12) that on any horizontal curve, $D S_{\gamma}^{\alpha \beta}=d S_{\gamma}^{\alpha \beta}$. Hence we get from (2.11) the following important

(2.16) COROLLARY. If the differential d is taken along a horizontal curve, then

$$
d S_{\gamma}^{\alpha \beta}=\left(\delta S_{k}^{i j}\right) \stackrel{\alpha}{\alpha \beta k} x_{i}^{\beta} x_{j}
$$

2.4. REMARK 1. In Theorem (2.4), we have a necessary and sufficient condition for a set of $n^{3}$ functions on $B$ to correspond to a tensor of type $(2,1)$ on $M$. When a linear connection $\gamma$ on $M$ has been given, there is also a theorem due to S. S. Chern [2, p. 79] which we state in a slightly different form as follows:

(2.17) Theorem. $A$ set of $n^{3}$ functions $S_{\gamma}^{\alpha \beta}$ on $B$ corresponds to a tensor of type $(2,1)$ on $M$ iff the $n^{3} 1$-forms

$$
\phi_{\gamma}^{\alpha \beta} \equiv d S_{\gamma}^{\alpha \beta}+\omega_{\epsilon}^{\alpha} S_{\gamma}^{\epsilon \beta}+\omega_{\epsilon}^{\beta} S_{\gamma}^{\alpha \epsilon}-\omega_{\gamma}^{e} S_{\epsilon}^{\alpha \beta}
$$

are all horizontal.

REMARK 2. The results of $\$ \S 2.1-2.3$ enable us to give an alternative definition to the covariant derivative of a tensor on $M$ when the linear connection is defined by a field of $n$-planes on $B$ as in (1.11).

Let $Y$ be any vector field on $M$. It is easy to prove that there is a unique horizontal vector field $\bar{Y}$ on $B$ such that $\pi^{\prime} \bar{Y}=Y$, where $\pi^{\prime}$ is the differential of the projection $\pi: B \rightarrow M$. In terms of local coordinates, if $Y=Y^{i}\left(\partial / \partial u^{j}\right)$, then (cf. (1.17))

$$
\bar{Y}=Y^{j}\left(\frac{\partial}{\partial u^{j}}-\Gamma_{j k}^{i} x_{\gamma}^{k} \frac{\partial}{\partial x_{\gamma}^{i}}\right) .
$$

Since $\bar{Y}$ is horizontal, we have by using Corollary (2.16) that

$$
\begin{aligned}
\bar{Y} S_{\gamma}^{\alpha \beta} & =\left\langle\bar{Y}, d S_{\gamma}^{\alpha \beta}\right\rangle=\left\langle\bar{Y},\left(\delta S_{k}^{i j}\right) x_{i}^{\alpha \beta x_{j}^{k} x_{\gamma}^{k}}\right\rangle \\
& =\left\langle Y,\left(\delta S_{k}^{i j}\right) x_{i}^{\alpha x_{j}^{k} x_{\gamma}^{k}}\right\rangle=\left(\nabla_{h} S_{k}^{i j}\right) Y^{h} x_{i}^{\alpha} x_{j}^{\beta} x_{\gamma}^{k} .
\end{aligned}
$$

Therefore, $\bar{Y} S_{\gamma}^{\alpha \beta}$ are the set of $n^{3}$ functions on $B$ corresponding to the tensor $\left(\nabla_{h} S_{k}^{i j}\right) Y^{h}$ on $M$. That $\bar{Y} S_{\gamma}^{\alpha \beta}$ should correspond to a tensor of type $(2,1)$ on $M$ can be verified directly by using the criterion given in Theorem (2.4). Hence, we obtain the following generalization of a result of Nomizu [4, p. 53] for the case of contravariant vector: 
(2.18) If a linear connection on $M$ is defined by a field of $n$-planes on $B$ as in (1.11), we may define the covariant derivative of a tensor $S$, say of type $(2,1)$, on $M$ by first constructing the functions $S_{\gamma}^{\alpha \beta}$ on $B$ corresponding to $S$ and then taking $\left(\nabla_{h} S_{k}^{i j}\right) Y^{h}$ to be the tensor on $M$ corresponding to the set of functions $\bar{Y} S_{\gamma}^{\alpha \beta}$ on $B$.

3.1. Recurrent tensors on a connected smooth manifold $M$. We assume for the remaining part of this paper that the smooth manifold $M$ is connected. This assumption has the following easily proved implications which we shall later use:

(3.1) Lemma. Any two points in $M$ can be joined by a sectionally smooth curve.

(3.2) Lemma. For any point $u_{1} \in M$ and any point $z_{0} \in B$, the two submanifolds $\pi^{-1}\left(u_{1}\right)$ and $B\left[z_{0}\right]=\left\{z \mid z \in B, z \sim z_{0}\right\}$ of $B$ have nonempty intersection.

(3.3) Lemma. For any two points $z_{0}, z_{1} \in B$, there exists an element $a \in G$ such that $B\left[z_{1}\right]=B\left[z_{0} a\right]$, i.e., $B\left[z_{1}\right]=\left\{z^{\prime} \mid z^{\prime}=z a, z \in B\left[z_{0}\right]\right\}$.

A tensor $S$ on $M$ is said to be recurrent (with respect to a given linear connection) if it is not a zero tensor and if its covariant derivative $\nabla S$ is equal to the tensor product of a covariant vector with $S$ itself. Expressed in terms of local coordinates $\left(U, u^{i}\right)$, this means that

$$
\nabla_{h} S_{k}^{i j \cdots}=W_{h} S_{k}^{i j \cdots}
$$

In particular, a tensor $S$ on $M$ is said to be covariantly constant if $S \neq 0$ and $\nabla S=0$.

We now prove

(3.4) THEOREM. If a tensor $S$ on $M$ is recurrent, then the restrictions of its corresponding functions $S_{\gamma}^{\alpha \beta} \ldots$ on $B$ to any (sectionally smooth) horizontal curve in $B$ are proportional to a set of constants.

Proof. Since any sectionally smooth curve in a smooth manifold may be regarded as one each of whose smooth sections lies in a coordinate neighborhood, it suffices to prove our theorem for the case of a smooth horizontal curve $z(\tau), 0 \leqq \tau \leqq 1$, in a coordinate neighborhood $\pi^{-1}(U) \subset B$. Let $u(\tau)=\pi z(\tau)$. Then $u(\tau)$ is a smooth curve in the coordinate neighborhood $U \subset M$. For simplicity, we take a tensor $S$ of type $(2,1)$ on $M$, and let $S_{\gamma}^{\alpha \beta}$ be its corresponding functions on $B$. Since all the 1 -forms $\omega_{\mu}^{\lambda}$ vanish along horizontal curves, it follows from Corollary (2.16) that on $z(\tau)$,

$$
\begin{aligned}
& \frac{d S_{\gamma}^{\alpha \beta}}{d \tau}=\left\langle\frac{d z}{d \tau}, d S_{\gamma}^{\alpha \beta}\right\rangle \\
& =\left\langle\frac{d z}{d \tau},\left(\delta S_{k}^{i j}\right) x_{i}^{\alpha} x_{j}^{\beta} x_{\gamma}^{k}\right\rangle=\left\langle\frac{d u}{d \tau}, \delta S_{k}^{i j}\right\rangle x_{i}^{\alpha} x_{j}^{\beta} x_{\gamma}^{k} .
\end{aligned}
$$


Hence

$$
\frac{d S_{\gamma}^{\alpha \beta}}{d \tau}=\left(\nabla_{h} S_{k}^{i j}\right) \frac{d u^{h}}{d \tau} \underset{x_{i} x_{j}^{\beta} x_{\gamma} x^{k}}{ }
$$

If $S$ is recurrent, we have $\nabla_{h} S_{k}^{\text {\}j }}=W_{h} S_{k}^{\text {ij }}$ and equation (3.5) becomes

$$
\frac{d S_{\gamma}^{\alpha \beta}}{d \tau}=\left(W_{h} \frac{d u^{h}}{d \tau}\right) S_{k}^{i j} x_{i}^{\alpha \beta} x_{j}^{\beta} x_{\gamma}
$$

i.e.,

$$
\frac{d S_{\gamma}^{\alpha \beta}}{d \tau}=f(\tau) S_{\gamma}^{\alpha \beta}
$$

where the function $f(\tau)=W_{h}(u(\tau)) d u^{h} / d \tau$ is the same for all the functions $S_{\gamma}^{\alpha \beta}$.

For a function $S_{\gamma}^{\alpha \beta}$ which has a zero at some point $z\left(\tau_{0}\right)$ of the curve $z(\tau)$, it follows from (3.6) and an existence theorem in differential equations that this $S_{\gamma}^{\alpha \beta}$ is always zero along the curve $z(\tau)$.

For a function $S_{\gamma}^{\alpha \beta}$ which has no zero on $z(\tau)$, equation (3.6) can be written as

$$
\frac{d \log S_{\gamma}^{\alpha \beta}}{d \tau}=f(\tau)
$$

Integration of this gives

$$
S_{\gamma}^{\alpha \beta}(z(\tau))=c_{\gamma}^{\alpha \beta} \tilde{g}(\tau), \quad\left(c_{\gamma}^{\alpha \beta}=\text { constant }\right),
$$

where $g(\tau)=\exp \left(\int f(\tau) d \tau\right)$ is a function which is nowhere zero on $z(\tau)$. Since the case when a function $S_{\gamma}^{\alpha \beta}$ is always zero on $z(\tau)$ can be regarded as a special case of (3.7) with $c_{\gamma}^{\alpha \beta}=0$, our theorem is proved by (3.7).

Any easy consequence of Theorem (3.4) is

(3.8) THEOREM. A recurrent tensor on a connected smooth manifold with a linear connection has no zero.

Proof. By definition, a recurrent tensor $S$ is not a zero tensor. Assume that $S$ has a zero at $u_{0} \in M$, and let $u_{1}$ be any other point of $M$. By Lemma (3.1), there exists in $M$ a sectionally smooth curve $u(\tau), 0 \leqq \tau \leqq 1$, joining $u(0)=u_{0}$ to $u(1)=u_{1}$. Let $z_{0}$ be any point in $B$ above $u_{0}$ (i.e., $\pi z_{0}=u_{0}$ ). Then by (1.13), there exists a unique lift $z(\tau)$ of $u(\tau)$ beginning at $z(0)=z_{0}$ and ending at $z(1)$ such that $\pi z(1)=u(1)=u_{1}$. Since $S\left(u_{0}\right)=0$ by assumption, $S_{\gamma}^{\alpha \beta}\left(z_{0}\right)$ are all zero. Therefore, by Theorem (3.4), $S_{\gamma}^{\alpha \beta}$ are all zero along $z(\tau)$, and in particular, 
$S_{\gamma}^{\alpha \beta}\left(z_{1}\right)$ are all zero. Hence $S\left(u_{1}\right)=0$ and so $S$ is a zero tensor. This contradiction to our assumption proves that $S$ has no zero.

We now prove our main

(3.9) TheOREM. Let $M$ be a connected smooth manifold with a linear connection. Then a tensor $S$ on $M$ is recurrent iff the restrictions of its corresponding functions on $B$ to any submanifold $B\left[z_{0}\right]=\left\{z \mid z \in B, z \sim z_{0}\right\}$ of $B$ have no common zero and are proportional to a set of constants.

Proof. Let $z$ be any point of $B\left[z_{0}\right]$. Then, there exists a sectionally smooth horizontal curve joining $z$ to $z_{0}$. Let $S$ be a recurrent tensor of type $(2,1)$ on $M$. Then by Theorem (3.8), $S$ has no zero (in $M$ ) and so the functions $S_{\gamma}^{\alpha \beta}$ have no common zero in $B\left[z_{0}\right]$. Furthermore, it follows from Theorem (3.4) that

$$
S_{\gamma}^{\alpha \beta}(z)=S_{\gamma}^{\alpha \beta}\left(z_{0}\right) g(z), \quad z \in B\left[z_{0}\right],
$$

where $g$ is some smooth function on $B\left[z_{0}\right]$. Therefore, the restrictions of $S_{\gamma}^{\alpha \beta}$ to $B\left[z_{0}\right]$ are proportional to a set of constants.

The converse is more difficult to prove. Let $S$ be any tensor of type $(2,1)$ on $M$ and $S_{\gamma}^{\alpha \beta}$ its corresponding functions on $B$. Assume that the restrictions of $S_{\gamma}^{\alpha \beta}$ to $B\left[z_{0}\right]$ have no common zero and are proportional to a set of constants. Then

$$
S_{\gamma}^{\alpha \beta}(z)=c_{\gamma}^{\alpha \beta} g(z), \quad \quad c_{\gamma}^{\alpha \beta}=\text { constant, } \quad z \in B\left[z_{0}\right],
$$

and the function $g(z)$ has no zero in $B\left[z_{0}\right]$.

Let $U$ be any coordinate neighborhood in $M$ and $u_{1}$ any point in $U$. By Lemma (3.2), there exists some point $z_{1} \in B\left[z_{0}\right]$ such that $\pi z_{1}=u_{1}$. Let $u(\tau)$, $0 \leqq \tau \leqq 1$, be any smooth curve in $U$ such that $u\left(\tau_{1}\right)=u_{1}, 0<\tau_{1}<1$, and let $z(\tau)$ be the smooth lift of $u(\tau)$ such that $z\left(\tau_{1}\right)=z_{1}$. Then since $z(\tau)$ is a horizontal curve, $z(\tau)$ lies in $\pi^{-1}(U) \cap B\left[z_{0}\right]$. Therefore, it follows from (3.5) and (3.10) that on $z(\tau)$,

$$
\left(\nabla S_{k}^{i j}\right) \frac{d u^{l}}{d \tau} \underset{x_{i}^{\alpha} x_{j}^{\beta} x_{\gamma}^{k}}{d \tau}=\frac{d S_{\gamma}^{\alpha \beta}}{d \tau}=c_{\gamma}^{\alpha \beta} \frac{d g}{d \tau}=S_{\gamma}^{\alpha \beta} \frac{d g}{d \tau} \frac{1}{g},
$$

i.e.,

$$
\frac{d u^{l}}{d \tau}\left(\nabla / S_{k}^{i j}\right)=f S_{k}^{i j}
$$

where $f=d \log g / d \tau$ is some function of $\tau$ which depends on the curve $u(\tau)$. Now take $u(\tau)$ to be each of the $n$ curves 


$$
u_{(h)}^{l}(\tau)=\delta_{h}^{l}\left(\tau-\tau_{1}\right)+\left(u_{1}\right)^{l} .
$$

Then $d u_{(h)}^{\cdot} / d \tau=\delta_{h}^{l}$, and it follows from (3.11) that

$$
\left(\nabla_{h} S_{k}^{i j}\right)\left(u_{1}\right)=W_{h}\left(u_{1}\right) S_{k}^{i j}\left(u_{1}\right) .
$$

Since $u_{1}$ is any point in $U$, we have thus shown that in each coordinate system $\left(U, u^{i}\right)$, there exists a set of $n$ functions $W_{h}$ such that

$$
\nabla_{h} S_{k}^{i j}=W_{h} S_{k}^{i j}
$$

We now proceed to prove that these $n$ functions $W_{h}$ are the components in $\left(U, u^{i}\right)$ of a covariant vector $W$ globally defined on $M$. When this is done, it follows from (3.12) that $\nabla S$ is equal to the tensor product of $W$ and $S$; in other words, $S$ is a recurrent tensor.

Let us denote by $u^{i^{*}}=u^{i^{*}}\left(u^{1}, \cdots, u^{n}\right)$ a change of coordinates in $U$, and by $W_{n^{*}}^{*}$ the set of $n$ functions defined in $\left(U, u^{i^{*}}\right)$ such that

$$
\nabla_{h^{*}} S_{k^{*}}^{i^{*} *^{*}}=W_{h^{*}}^{*} S_{k^{*}}^{i^{*} *^{*}}
$$

From (3.12), $\left(3.12^{*}\right)$ and the fact that $\nabla S$ is a tensor on $M$, it follows that

$$
W_{h^{*}}^{*} S_{k^{*}}^{i^{*} j^{*}}=\frac{\partial u^{h}}{\partial u^{h^{*}}} \frac{\partial u^{i^{*}}}{\partial u^{i}} \frac{\partial u^{i^{*}}}{\partial u^{j}} \frac{\partial u^{k}}{\partial u^{k^{*}}} W_{h} S_{k}^{i j} .
$$

Since $S$ is a tensor on $M$, the above equation can be written as

$$
W_{h^{*}}^{*} S_{k^{*}}^{i^{*} j^{*}}=\frac{\partial u^{h}}{\partial u^{h^{*}}} W_{h} S_{k^{*}}^{i^{*}} \text {. }
$$

But by hypothesis, the functions $S_{\gamma}^{\alpha \beta}$ have no common zero in $B\left[z_{0}\right]$, so $S$ has no zero in $M$. Therefore, at each point of $U$, at least one of the components $S_{k^{*}}^{* * *}$ is not zero. Hence, it follows from (3.13) that the equation

$$
W_{h^{*}}^{*}=\frac{\partial u^{h}}{\partial u^{h^{*}}} W_{h}
$$

holds at each point of $U$ and so everywhere in $U$. In other words, $W_{h}$ are the components of a covariant vector $W_{U}$ on $U$.

Now let us regard the $u^{i^{*}}$ in the preceding paragraph as the local coordinates in another neighborhood $U^{*}$ and let $W_{U^{*}}$ be the covariant vector defined on $U^{*}$. Then by the same arguments, we may conclude that equation (3.14) holds on $U \cap U^{*}$. This means that $W_{U^{*}}=W_{U}$ on $U \cap U^{*}$. In other words, the covariant vectors $W_{U}$ which we have determined, one for each coordinate neighborhood, piece together to form a covariant vector $W$ globally defined on $M$. Hence our theorem is completely proved. 
REMARK. Both the statement of Theorem (3.9) and its proof imply that if the restrictions of the functions $S_{\gamma}^{\alpha \beta}$ to some $B\left[z_{0}\right]$ are proportional to a set of constants, their restrictions to any other $B\left[z_{1}\right]$ are also proportional to a set of constants. This can be seen directly as follows. By Lemma (3.3), there exists an element $a \in G$ such that

$$
B\left[z_{1}\right]=B\left[z_{0} a\right]=\left\{z^{\prime} \mid z^{\prime}=z a, z \in B\left[z_{0}\right]\right\} .
$$

If

$$
S_{\gamma}^{\alpha \beta}(z)=c_{\gamma}^{\alpha \beta} g(z), \quad \quad c_{\gamma}^{\alpha \beta}=\text { const., } \quad z \in B\left[z_{0}\right],
$$

then for $z^{\prime} \in B\left[z_{1}\right]$,

$$
S_{\gamma^{\prime}}^{\alpha^{\prime} \beta^{\prime}}\left(z^{\prime}\right)=S_{\gamma^{\prime}}^{\alpha^{\prime} \beta^{\prime}}(z a)=S_{\gamma}^{\alpha \beta}(z) \tilde{a}_{\alpha}^{\alpha^{\prime}} \tilde{a}_{\beta}^{\beta^{\prime}} a_{\gamma^{\prime}}^{\gamma}=c_{\gamma}^{\alpha \beta} \tilde{a}_{\alpha}^{\alpha^{\prime}} \tilde{a}_{\beta}^{\beta^{\prime}} a_{\gamma^{\prime}}^{\gamma} g(z) .
$$

Therefore,

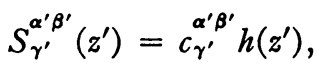

$$
\begin{aligned}
& z^{\prime} \in B\left[z_{1}\right],
\end{aligned}
$$

where $h\left(z^{\prime}\right)=g\left(z^{\prime} a^{-1}\right)$, and $c_{\gamma}^{\alpha^{\prime} \beta^{\prime}}=c_{\gamma^{\prime}}^{\alpha \beta} a_{\alpha}^{\alpha^{\prime}} \tilde{a}_{\beta}^{\beta^{\prime}} a_{\gamma^{\prime}}^{\gamma}$ are again a set of constants.

3.2. Covariantly constant tensors on a connected smooth manifold. If $S$ is a covariantly constant tensor of type $(2,1)$ on $M$, equations $(3.5)$ become $d S_{\gamma}^{\alpha \beta} / d \tau=0$. Therefore, $S_{\gamma}^{\alpha \beta}$ are all constant on any horizontal curve, and consequently are all constant on $B\left[z_{0}\right]$. Conversely, let $S$ be a tensor of type $(2,1)$ on $M$ such that its corresponding functions $S_{\gamma}^{\alpha \beta}$ are all constant on $B\left[z_{0}\right]$. Since $M$ is connected, on account of Lemma (3.2), any smooth curve $u(\tau)$ in $M$ has some smooth lift $z(\tau)$ lying in $B\left[z_{0}\right]$, so that on $z(\tau)$, the functions $S_{\gamma}^{\alpha \beta}$ are all constant. Then it follows from (3.5) that

$$
\frac{d u^{h}}{d \tau}\left(\nabla_{h} S_{k}^{i j}\right)=0
$$

for any smooth curve $u(\tau)$ in $M$. This means that $\nabla_{h} S_{k}^{y}=0$. Hence we have proved

(3.15) THEOREM. A tensor on a connected smooth manifold $M$ with a linear connection is covariantly constant iff its corresponding functions on $B$ are all constant (but not all zero) on any $B\left[z_{0}\right]$.

A special case of Theorem (3.15) was proved by S. Kobayashi [3, p. 31].

4.1. Linear connection with recurrent curvature or recurrent torsion. When a linear connection $\gamma$ on $M$ is given, $\gamma$ is said to be of recurrent curvature (or of recurrent torsion) if the curvature tensor $R$ (or the torsion tensor $T$ ) is recurrent. For a local theory of linear connections with recurrent curvature and zero torsion, see Wong [5]. 
We see from (1.15) that the functions on $B$ which correspond to the curvature tensor $R$ are the functions $R_{\mu \alpha \beta}^{\lambda}$. By Theorem (3.9), $R$ is recurrent iff the restrictions of these functions to any $B\left[z_{0}\right]$ have no common zero and are proportional to a set of constants, i.e., iff

$$
R_{\mu \alpha \beta}^{\lambda}(z)=\stackrel{\lambda}{c_{\mu \alpha \beta}} g(z) \text { on } B\left[z_{0}\right] \text {, }
$$

where $c_{\mu \alpha \beta}^{\lambda}=$ constant, and the function $g(z)$ has no zero on $B\left[z_{0}\right]$. On the other hand, we have from (1.19) $)_{3}$ that

$$
v\left[E_{\alpha}, E_{\beta}\right](z)=-R_{\mu \alpha \beta}^{\lambda}(z) E_{\lambda}^{\mu}(z) \text { on } B,
$$

where the left side is the vertical component of the vector field $\left[E_{\alpha}, E_{\beta}\right]$. Thus, $R$ is recurrent iff

$$
v\left[E_{\alpha}, E_{\beta}\right](z)=-g(z)\left(c_{\mu \alpha \beta}^{\lambda} E_{\lambda}^{\mu}(z)\right) \text { on } B\left[z_{0}\right] \text {, }
$$

where the function $g(z)$ has no zero on $B\left[z_{0}\right]$. Let us say that two vector fields $X$ and $Y$ on a differentiable manifold are co-directional if there exists a function $g$ having no zero such that $Y=g X$. Then (4.1) expresses the fact that the restriction of the vector field $v\left[E_{\alpha}, E_{\beta}\right]$ to $B\left[z_{0}\right]$ is co-directional with a fundamental vector field. A similar result can be obtained for the case of recurrent torsion. We state these results in the following

(4.2) THEOREM. Let $M$ be a connected smooth manifold with a linear connection $\gamma$. Let $B$ be the frame bundle over $M, E_{\alpha}(1 \leqq \alpha, \beta \leqq n)$ the basic vector fields on $B, v\left[E_{\alpha}, E_{\beta}\right]$ and $h\left[E_{\alpha}, E_{\beta}\right]$ the vertical and horizontal components of the vector field $\left[E_{\alpha}, E_{\beta}\right]$. Let $z_{0}$ be any point in $B$ and let $B\left[z_{0}\right]=\left\{z \mid z \in B, z \sim z_{0}\right\}$. Then $\gamma$ is of recurrent curvature iff the restriction of each $v\left[E_{\alpha}, E_{\beta}\right]$ to $B\left[z_{0}\right]$ is co-directional with a fundamental vector field. And $\gamma$ is of recurrent torsion iff the restriction of each $h\left[E_{\alpha}, E_{\beta}\right]$ to $B\left[z_{0}\right]$ is co-directional with a basic vector field.

A similar argument based on Theorem (3.15) and formulas (1.19) ${ }_{3}$ leads easily to the following theorem of Nomizu's [4, p. 73].

(4.3) Theorem (Nomizu). The notation being as in Theorem (4.1), then the curvature tensor of $\gamma$ is covariantly constant iff the restriction of each $v\left[E_{\alpha}, E_{\beta}\right]$ to $B\left[z_{0}\right]$ is a fundamental vector field. And the torsion tensor of $\gamma$ is covariantly constant iff the restriction of each $h\left[E_{\alpha}, E_{\beta}\right]$ to $B\left[z_{0}\right]$ is a basic vector field.

4.2. The holonomy algebra of a linear connection with recurrent curvature. An interpretation in terms of the so-called holonomy algebra can also be given to our result that for a linear connection with recurrent curvature, the restrictions of the functions $R_{\mu \alpha \beta}^{\lambda}$ to $B\left[z_{0}\right]$ are proportional to a set of constants. By definition, the holonomy algebra of a linear connection is the Lie 
algebra of the holonomy group. Let $z \in B$, and $Z_{1}, Z_{2}$ be any two horizontal vectors at $z$. If we regard the $n \times n$ matrix with elements $\Omega_{\mu}^{\lambda}\left(Z_{1}, Z_{2}\right)(z)$ as an element of the Lie algebra of $G=G L(n, R)$, then according to a theorem due to E. Cartan, Ambrose and Singer [1] (also Nomizu [4, pp. 39-41]), we know that the holonomy algebra (or rather, the Lie algebra of the holonomy group with reference point $z_{0} \in B$ ) is a sub-algebra of the Lie algebra of $G$ spanned by the elements $\Omega_{\mu}^{\lambda}\left(Z_{1}, Z_{2}\right)(z)$ when $z$ runs through the point set $B\left[z_{0}\right]$, and $Z_{1}, Z_{2}$ run through any set of linearly independent horizontal vectors at $z$.

Now $\Omega_{\mu}^{\lambda}=(1 / 2) R_{\mu \alpha \beta}^{\lambda} \theta^{\alpha} \wedge \theta^{\beta}$. Let us take as the set of linearly independent horizontal vectors at $z$ the vectors of the basic vector fields $E_{\alpha}$. Then since $\left\langle\theta^{\beta}, E_{\alpha}\right\rangle=\delta_{\alpha}^{\beta}$, we have

$$
\begin{aligned}
\left\{\Omega_{\mu}^{\lambda}\left(E_{\alpha}, E_{\beta}\right)\right\}(z) & =\left\{(1 / 2) R_{\mu \rho \rho}^{\lambda} \theta^{\rho} \wedge \theta^{\sigma}\left(E_{\alpha}, E_{\beta}\right)\right\}(z) \\
& =(1 / 2) R_{\mu \alpha \beta}^{\lambda}(z), \quad z \in B .
\end{aligned}
$$

Therefore, the holonomy algebra is spanned by the elements

$$
R_{\mu \alpha \beta}^{\lambda}(z),
$$$$
1 \leqq \alpha, \beta \leqq n,
$$

of the Lie algebra of $G L(n, R)$ when $z$ runs through the point set $B\left[z_{0}\right]$.

If the linear connection $\gamma$ is of recurrent curvature,

$$
R_{\mu \alpha \beta}^{\lambda}(z)=\stackrel{\lambda}{c_{\mu \alpha \beta} g} g(z),
$$

where $c_{\mu \alpha \beta}^{\lambda}=$ constant, $z \in B\left[z_{0}\right]$, and the function $g(z)$ has no zero on $B\left[z_{0}\right]$. Therefore, for any fixed $\alpha$ and $\beta$, the element $R_{\mu \alpha \beta}^{\lambda}(z)$ at any two points of $B\left[z_{0}\right]$ are co-directional. Hence

(4.3) THEOREM. Let $M$ be a connected smooth manifold of dimension $n$ with a linear connection $\gamma$. If $\gamma$ is of recurrent curvature, then the holonomy algebra is spanned by the elements

$$
R_{\mu \alpha \beta}^{\lambda}(z), \quad 1 \leqq \alpha, \beta \leqq n,
$$

of the Lie algebra of $G L(n, R)$, where $z$ is any fixed point in the frame bundle $B$ over $M$, this Lie sub-algebra being the same for all the points of $B$ which can be joined to $z$ by (sectionally smooth) horizontal curves.

Since $R_{\mu \alpha \beta}^{\lambda}$ is skew-symmetric in the indices $\alpha, \beta$, there are at most $n(n-1) / 2$ such elements. Therefore,

(4.4) Corollary. On a connected smooth manifold of dimension $n$, the holonomy group of a linear connection with recurrent curvature is at most of dimension $n(n-1) / 2$. 
Added in proof. Theorems (3.8), (3.9) and (4.3) greatly facilitate the study of linear connections with recurrent curvature and zero torsion. Many results have been obtained which will be published in a forthcoming paper.

\section{REFERENCES}

1. W. Ambrose and I. Singer, $A$ theorem on holonomy, Trans. Amer. Math. Soc. vol. 75 (1953) pp. 428-443.

2. S. S. Chern, Differentiable manifolds, mimeographed notes, University of Chicago, 1959.

3. S. Kobayashi, Espaces d connexions affines et riemanniennes symetriques, Nagoya Math. J. vol. 9 (1955) pp. 25-37.

4. K. Nomizu, Lie groups and differential geometry, Mathematical Society of Japan, 1956.

5. Yung-Chow Wong, A class of non-Riemannian $K^{*}$-spaces, Proc. London Math. Soc., 3rd series, vol. 3 (1953) pp. 118-128.

6. - Linear connections and quasi-connections of a differentiable manifold, to appear.

UNIVERSITY OF HoNg Kong,

Hong Kong 